\title{
Liquefaction of Highly Saturated Sand Layer under Oscillating Water Pressure
}

\author{
Hiroshi NAG $\bar{O}^{*}$ \\ (Received September 11,1981)
}

Synopsis

In this paper the vertical distribution of pore water pressure in the highly saturated sand layer under the oscillating water pressure are treated theoretically and experimentally.

By the experiments it is shown that the water pressure acting on the sand surface propagates into the sand layer with the damping in amplitude and the lag in phase, and that the liquefaction, the state that the effective stress becomes zero, occurs under certain conditions. These experimental results are explained fairly well by the same theoretical treatment as for the ground water problems in the elastic aquifer.

The main characteristics of liquefaction clarified by the analysis are as follows.

1) The depth of the liquified layer increases with the increase of the amplitude and the frequency of the oscillating water pressure.

2) The increase of the volume of the water and the air in the layer increases the liquified depth. Especially the very small amount of the air affects the liquefaction significantly.

3) The liquified depth decrease rapidly with the increase of the compressibility coefficient of the sand. 4) In the range beyond a certain value of the permeability coefficient the liquified depth decrease with the increase of the coefficient.

* Department of Civil Engineering 


\section{Introduction}

Under the oscillating water pressure normal to the surface of the highly saturated sand layer the pore water pressure in the layer changes with time, and according to circumstances the excess pore pressure occurs. The increase of the excess. pore pressure brings to the decrease of the effective stress. Then, under certain conditions it is expected that the effective stress becomes to be equal to zero, that is, the state of liquefaction occurs. The liquified sand is removed easily by the flow tangential to the surface, and the sand layer will be scoured successively. Therefore, the clarification of the dynamical mechanism of such liquefaction is very important to design of hydraulic structures arounded by the sand layer.

In this report as an basic study for the explanation of the local scouring mechanism around the hydraulic structures and for the establishment of the prevention works against the scouring the influence of the properties of the oscillating water pressure and the sand layer on the characteristics of the liquefaction is investigated theoretically and experimentally.

2. Derivation of fundamental equation for pore water pressure

In the theoretical treatment we consider the vertically one-dimensional sand layer as shown in Fig.l. The layer is highly saturated with water and is placed on the impermeable bed. The depth of the layer is $D$. The oscillating water pressure $\rho g h_{\mathrm{S}}$ acts on the surface of the layer.

The motion of the water and the sand in the layer are analized by the same method as for the ground water problems in the elastic aquifer ${ }^{1)}, 2$ ). That is, it is assumed that the sand and the water are compressible, and then that the density of water, the porosity of the sand and the thickness of the layer are variable. But lateral strain of the sand is assumed to be negligible. The void in the layer is occupied by the water and the air. Then, it is assumed that the porosity $\lambda$ is composed of the part for the water $\lambda_{W}$ and the 
part for the air $\lambda_{a}$.

We consider the flow of water through the faces of an elemental volume as shown in Fig.1. By the law of conservation of matter the net inward flux must equal the rate at which water is accumulating within that volume. That is,

$$
\begin{aligned}
& \frac{\partial}{\partial t}\left(\rho \lambda_{W} \delta y \delta A\right) \\
& =-\frac{\partial}{\partial y}(\rho \cup \delta A) \delta y
\end{aligned}
$$

(1) Fig. I Sand layer under Oscillating water

where, $p:$ density of water, pressure

$U$ : velocity of flow,

$\delta A$ : horizontal cross sectional area.

The left hand side of the above equation is transformed as follows,

$$
\frac{\partial}{\partial t}\left(\rho \lambda_{W} \delta y \delta A\right)=\left(\lambda_{W} \frac{\partial \rho}{\partial t} \delta y+\rho \frac{\partial \lambda_{W}}{\partial t} \delta y+\rho \lambda_{W} \frac{\partial \delta y}{\partial t}\right) \delta A
$$

The vertical dimension of the elemental volume $\delta y$ varies with the vertical component of compressive stress $\sigma_{y}$ in accordance with,

$$
d(\delta y)=-\alpha \delta y d \sigma_{y}
$$

That is,

$$
\frac{\partial(\delta y)}{\partial t}=-\alpha \delta y \frac{\partial \sigma y}{\partial t}
$$

where, $\alpha$ : vertical compressibility of the sand.

Since the compressibility of the individual sand grains is small compared to the compressibility of the water and the change in the porocity, it will be permissible to consider that the volume of solid material in the volume 
remains constant. That is,

$$
\left\{1-\left(\lambda_{\mathrm{W}}+\lambda_{\mathrm{a}}\right)\right\} \delta \mathrm{y} \delta \mathrm{A}=\text { constant. }
$$

Differentiating the above equation by time variable $t$, we obtain the following equation.

$$
\frac{\partial\left(\lambda_{\mathrm{W}}+\lambda_{\mathrm{a}}\right)}{\partial t}=-\alpha\left\{1-\left(\lambda_{\mathrm{W}}+\lambda_{\mathrm{a}}\right)\right\} \frac{\partial \sigma_{\mathrm{y}}}{\partial t}
$$

If the inertia force of the sand is negligible small, it is permissible to consider that the vertical component of compressive stress and the pore water pressure can be equated to the downward acting force on the plane of contact of the sand. That is,

$$
\sigma_{\mathrm{y}}+\rho g h=\gamma_{\mathrm{S}} \mathrm{y}+\rho g \mathrm{~h}_{\mathrm{S}}
$$

The pore water pressure $\rho g h$ and the weight of the sand column above the plane of contact $\gamma_{\mathrm{s}} \mathrm{y}$ are represented as follows.

$$
\begin{aligned}
& \rho g h=\rho g\left(h_{S}+y+h^{\prime}\right), \\
& \gamma_{S} y=\rho_{S} g y(I-\lambda)+\rho g y \lambda_{W}
\end{aligned}
$$

where, $h^{\prime}$ : excess pore water pressure,

$\rho_{S} g$ : weight of unit volume of the individual sand grain,

g: accelaration due to qravity.

Substituting Eqs.(7), (8) into Eq.(6), and considering $\lambda_{\mathrm{W}} \fallingdotseq \lambda$,

$$
\sigma_{y}+\rho g h^{\prime}=\left(\rho_{s}-\rho\right) \operatorname{gy}(1-\lambda)=\text { constant }
$$

Accordingly,

$$
\rho g \frac{\partial h^{\prime}}{\partial t}=-\frac{\partial \sigma y}{\partial t}
$$

Then, the following equation is obtained from Eq. (4) and Eg. (10). 


$$
\frac{\partial \delta \mathrm{y}}{\partial t}=\rho g \alpha \delta \mathrm{y} \frac{\partial \mathrm{h}^{\prime}-}{\partial t}
$$

From Eq.(5) and Eq.(10),

$$
\frac{\partial\left(\lambda_{W}+\lambda_{a}\right)}{\partial t}=\rho g \alpha\left\{1-\left(\lambda_{W}+\lambda_{a}\right)\right\} \frac{\partial h^{\prime}}{\partial t}
$$

It is assumed that the volume of the air in the layer changes in accordance with Boyle's law. That is,

$$
\lambda_{\mathrm{a}} \delta \mathrm{y} \delta \mathrm{A}\left(\rho \mathrm{gh}+\mathrm{p}_{0}\right)=\bar{\lambda}_{\mathrm{a}} \delta \mathrm{y} \delta \mathrm{A}\left(\rho \mathrm{g} \overline{\mathrm{h}}+\mathrm{p}_{0}\right)=\text { constant },
$$

where, po: atmospheric pressure,

$\bar{\lambda}_{a}$ : porosity occupied by the air under the standard pressure $\left(\rho g \bar{h}+p_{0}\right)$.

Differentiating Eq.(13) by t, and using Eq.(10), the following equation is obtained.

$$
\frac{\partial \lambda_{a}}{\partial t}=-\frac{\lambda_{a}}{\left(h+p_{0} / \rho g\right)} \frac{\partial h}{\partial t}-\rho g \alpha \lambda_{a} \frac{\partial h^{\prime}}{\partial t}
$$

From Eqs. (12), (14),

$$
\frac{\partial \lambda_{W}}{\partial t}=\rho g \alpha\left(1-\lambda_{W}\right) \frac{\partial h^{\prime}}{\partial t}+\frac{\lambda_{a}}{\left(h+p_{0} / \rho g\right)} \frac{\partial h}{\partial t}
$$

The density of water changes in accordance with the following equation,

$$
\frac{d \rho}{\rho}=\beta d p, \quad p=\rho g h+p_{0}
$$

where, $\beta$ : compressibility of water,

p: absolute pressure.

Accordingly,

$$
\frac{\partial \rho}{\partial t}=\beta \rho^{2} g \frac{\partial h}{\partial t}
$$

Substituting Eqs.(11), (15), (17) into Eq.(2), 


$$
\frac{\partial}{\partial t}\left(\rho \lambda_{W} \delta y \delta A\right)=\rho^{2} g\left\{\left(B \lambda_{W}+\frac{\lambda_{a}}{\rho g h+p_{0}}\right) \frac{\partial h^{\prime}}{\partial t}+\alpha \frac{\partial h^{\prime}}{\partial t}\right\} \delta y \delta A
$$

It is assumed that the velocity of water in the layer is represented by Darcy's law,

$$
u=-k \frac{\partial h^{\prime}}{\partial y}
$$

where, $k$ : permeability coefficient.

Substituting Eqs.(18), (19) into eq.(1), finally we obtain the fundamental equation for the water pressure in the layer as follows.

$$
\begin{aligned}
\left(\beta \lambda_{W}+\frac{\lambda_{a}}{\rho g h+p_{0}}+\alpha\right) \frac{\partial h^{\prime}}{\partial t} & +\left(\beta \lambda_{W}+\frac{\lambda_{a}}{\rho g h+p_{0}}\right) \frac{\partial h_{S}}{\partial-} \\
& =\frac{k}{\rho g} \frac{\partial^{2} h^{\prime}}{\partial y^{2}}
\end{aligned}
$$

By solving the above equation under the following boundary conditions, the pore water pressure and the effective stress in the sand layer are obtained.

$$
\left.\begin{array}{lll}
\mathrm{h}^{\prime}=0 & \text { at } & \mathrm{y}=0 \\
\frac{\partial \mathrm{h}^{\prime}}{\partial \mathrm{y}}=0 & \text { at } & \mathrm{y}=\mathrm{D}
\end{array}\right\}
$$

The liquefaction occurs under the following condition,

$$
\frac{\sigma_{\mathrm{y}}}{\left(\rho_{\mathrm{S}}-\rho\right) \operatorname{gy}(I-\lambda)}=0
$$

For the case that the water pressure acting on the surface changes sinusoidally, that is,

$$
h_{S}=h_{0}+a_{0} \sin 2 \pi f t,
$$

Eq. (20) becomes,

$$
\begin{gathered}
\left(\beta \lambda_{W}+\frac{\lambda_{a}}{\rho g h+p_{0}}+\alpha\right) \frac{\partial h^{\prime}}{\partial t}+2 \pi f a_{0}\left(\beta \lambda_{W}+\frac{\lambda_{a}}{\rho g h+p_{0}}\right) \cos 2 \pi f^{\prime} t \\
=\frac{k}{\rho g} \frac{\partial^{2} h^{\prime}}{\partial y^{2}}
\end{gathered}
$$


where, $h_{0}$ : head of mean water pressure on the sand surface, $a_{0}$ : amplitude of oscillating water pressure, $f$ : frequency of oscillating water pressure,

Eq.(20) and Eq.(24) are nonlinear differential equations. Since it is difficult to solve these equations analytically, generally they are solved numerically.

\section{Experiments on liquefaction}

\subsection{Experimental procedure}

For the experiment the vertical cylinder as shown in Fig.2 was used. The inside diameter of the cylinder is $8.9 \mathrm{~cm}$. Its height is $1.5 \mathrm{~m}$. It is filled with the highly saturated sand to about $43 \%$ of its height. The water depth from the sand surface is about $80 \mathrm{~cm}$. The periodically oscillating air pressure acts on the water surface. Its amplitude is $95 \mathrm{~cm}$ in water head. The frequency changes from $1.37 \mathrm{~Hz}$ to $5.66 \mathrm{~Hz}$. The nearly uniform sand which $50 \%$ grain size is $0.25 \mathrm{~mm}$ was used. Two kinds of the sand sample concerning to the volume of the air in the layer was used. That is, the one sample which $\bar{\lambda}_{a}$ is about $0.5 \%$ at $\bar{h}=0.3 \mathrm{~m}$ and the another which $\bar{\lambda}_{a}$ is very small comparing with the former sample. In the following description each sample is called the aerated and the deaerated sample. The value of $\lambda_{W}$ is about $40 \%$. The permeability coefficient is about $2 \times 10^{-2} \mathrm{~cm} / \mathrm{s}$. The water pressure in the water and sand layer are picked up by the semi-conductor pressure transducer attached to the side of the cylinder.

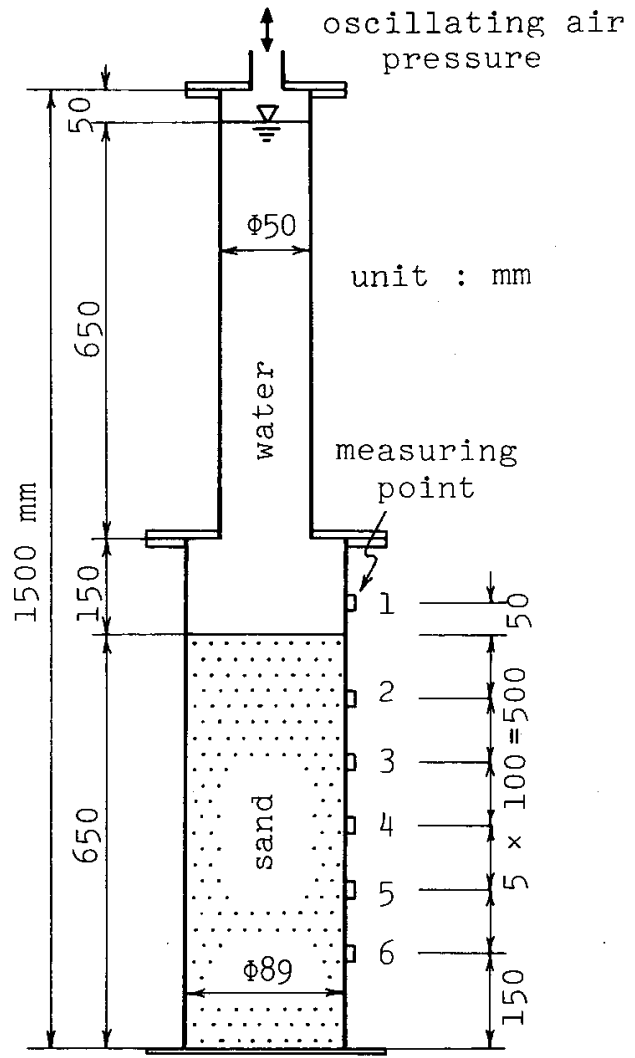

Fig.2 Model in experiments 


\subsection{Experimental results}

In Fig.3 (a), (b) the changes of the pore water pressure for the deaerated and the aerated sands are shown. These figures show that the water pressure on the sand surface propagates into the sand layer accompanied with the damping in amplitude and the lag in phase. The rate of the damping and the lag in the aerated sand is larger than that in the deaerated one.

Fig.4 (a), (b) show the damping ratio of the amplitude for both samples. The symbol $a$ in the figures represents the amplitude in the sand layer. These figures show that the amplitude decreases with increasing frequency.

Fig.5 (a), (b) show the change of the effective stress. It is shown in these figures that the state of the liquefaction $\left(\sigma_{\mathrm{y}}=0\right)$ appears at the point 1 for the deaerated sample and at all measuring points for the aerated one.

Figs.6, 7 and 8 show the calculated curves of the pore water pressure, the damping ratio and the effective stress corresponding to Figs. 3, 4 and 5 respectively. In the calculation it is assumed that the oscillating water pressure on the sand surface changes sinusoidally, and that the value of the compressibility of the sand $\alpha$ is $1.8 \times 10^{-6} \mathrm{~cm}^{2} / \mathrm{g}$. Comparing with experimental results, the calculated curves explain the experimental results fairly well. The differences between the experimental values and the calculated curves are considered to be mainly due to the approximation of the oscillating water pressure and the compressibility in the calculation.

4. Characteristics of liquefaction

\section{I Factors affecting liquefaction}

The characteristics of the liquefaction depend on the properties of water, sand layer, oscillating water pressure and the field. For convenience adopting the sinusoidal excitation like Eq.(23) as the oscillating water pressure, the characteristic parameters influencing on the phenomenon are as 


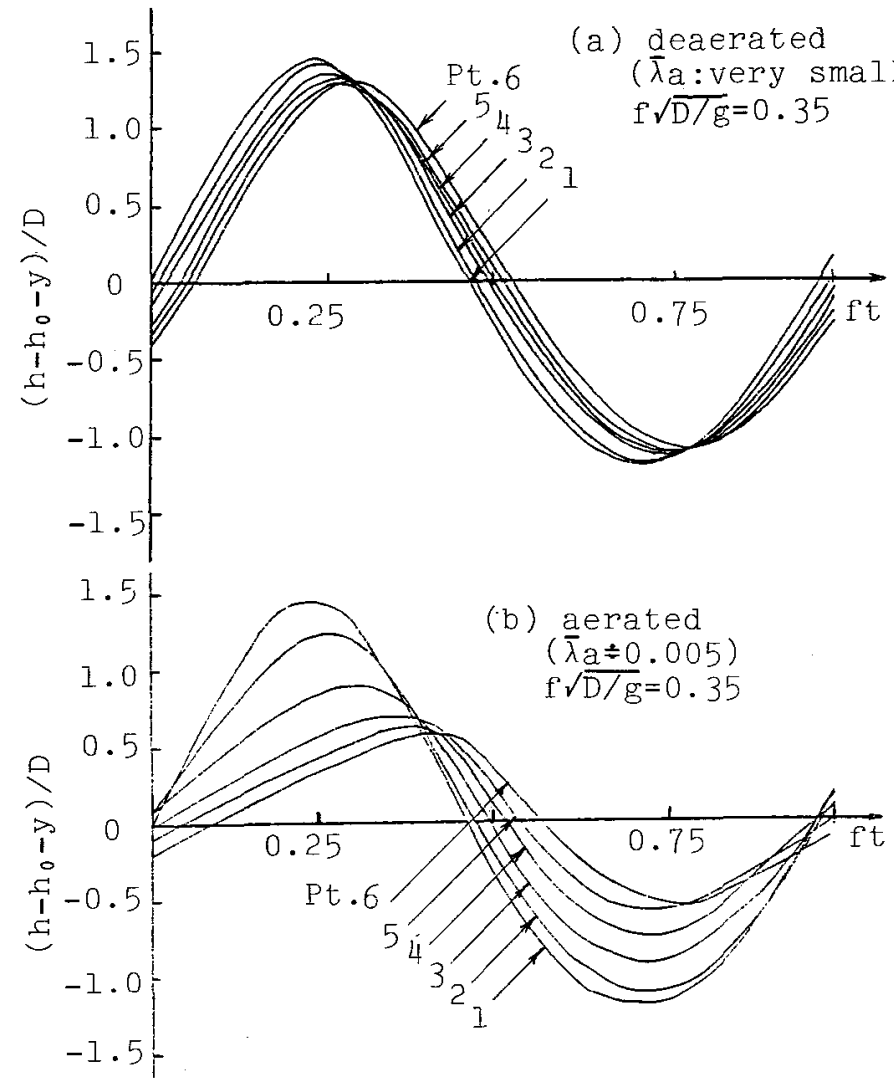

Fig. 3 Pore water pressure (observed)
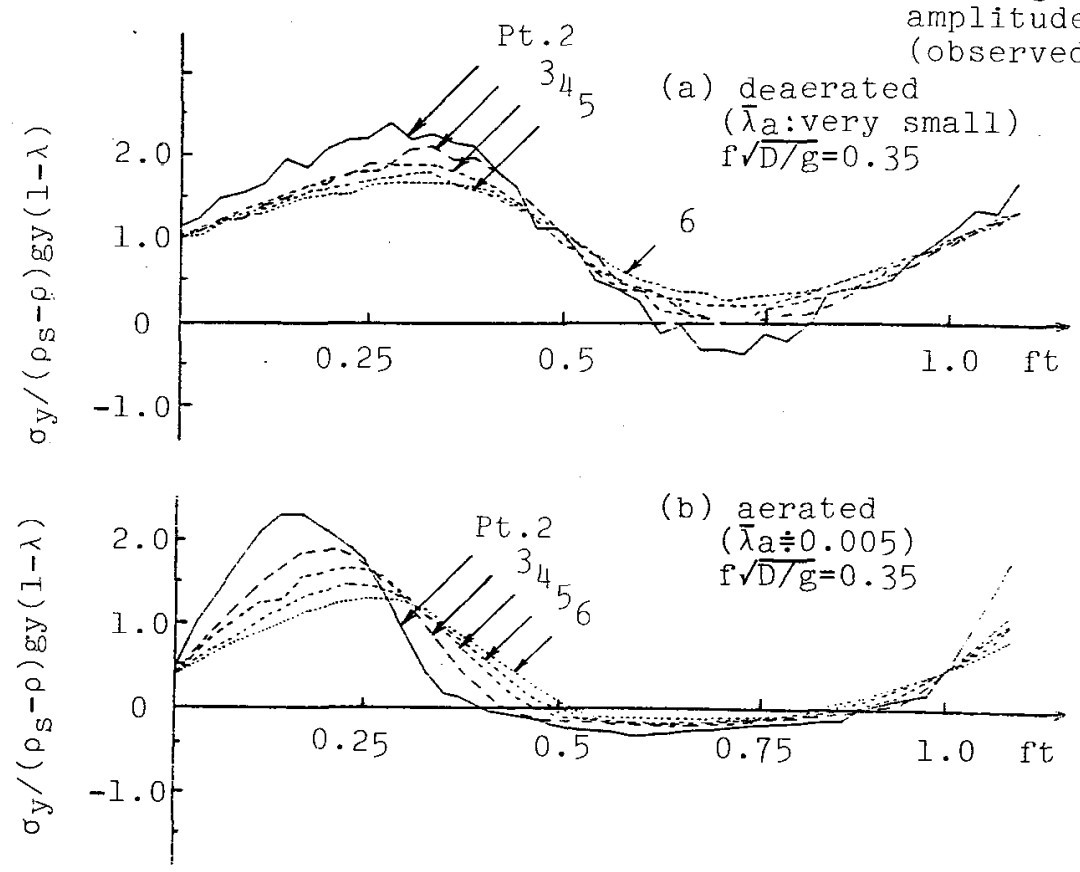

Fig. 5 Effective stress (observed) 

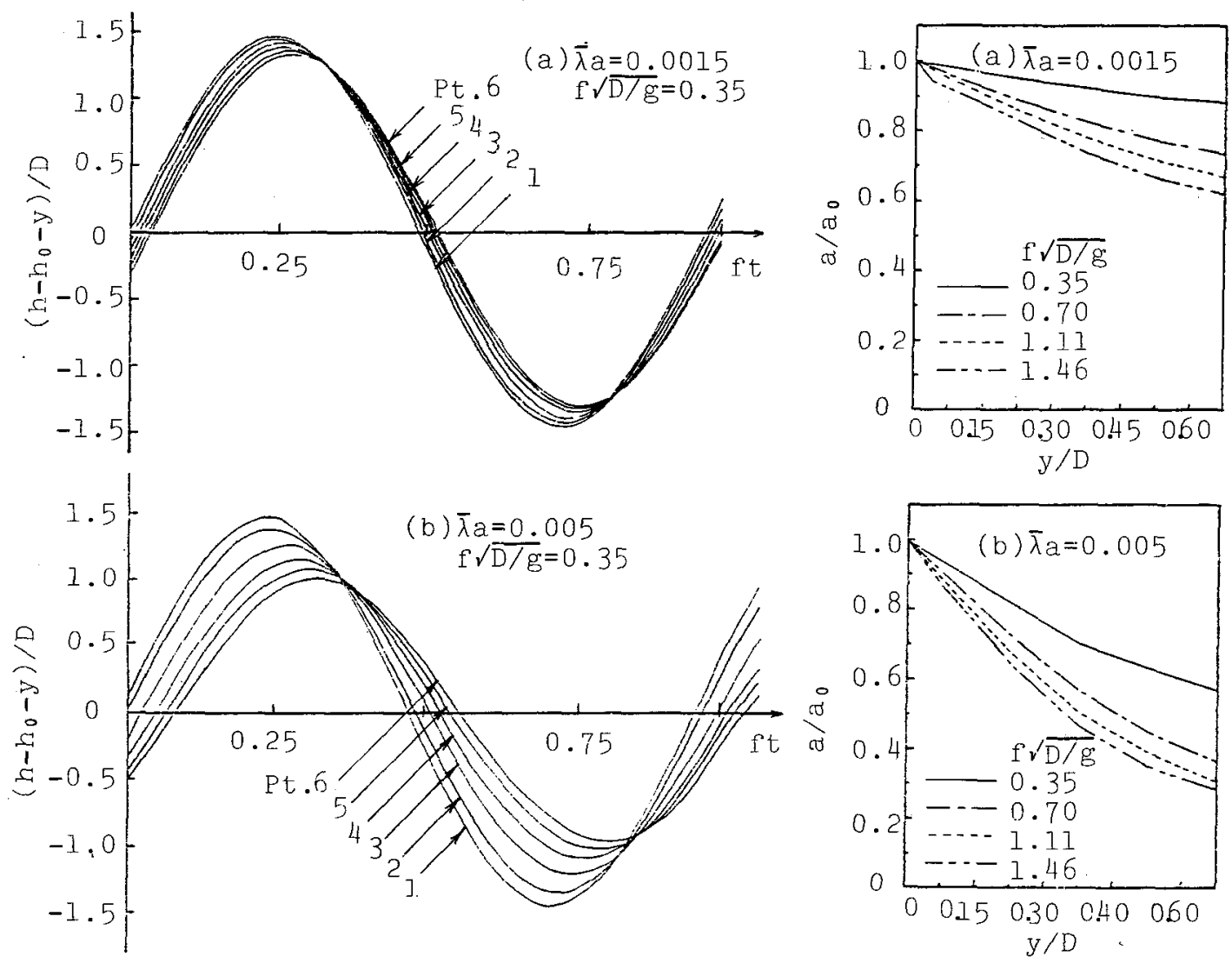

Fig. 6 Pore water pressure (calculated)

Fig.7 Damping ratio of amplitude (calculated)
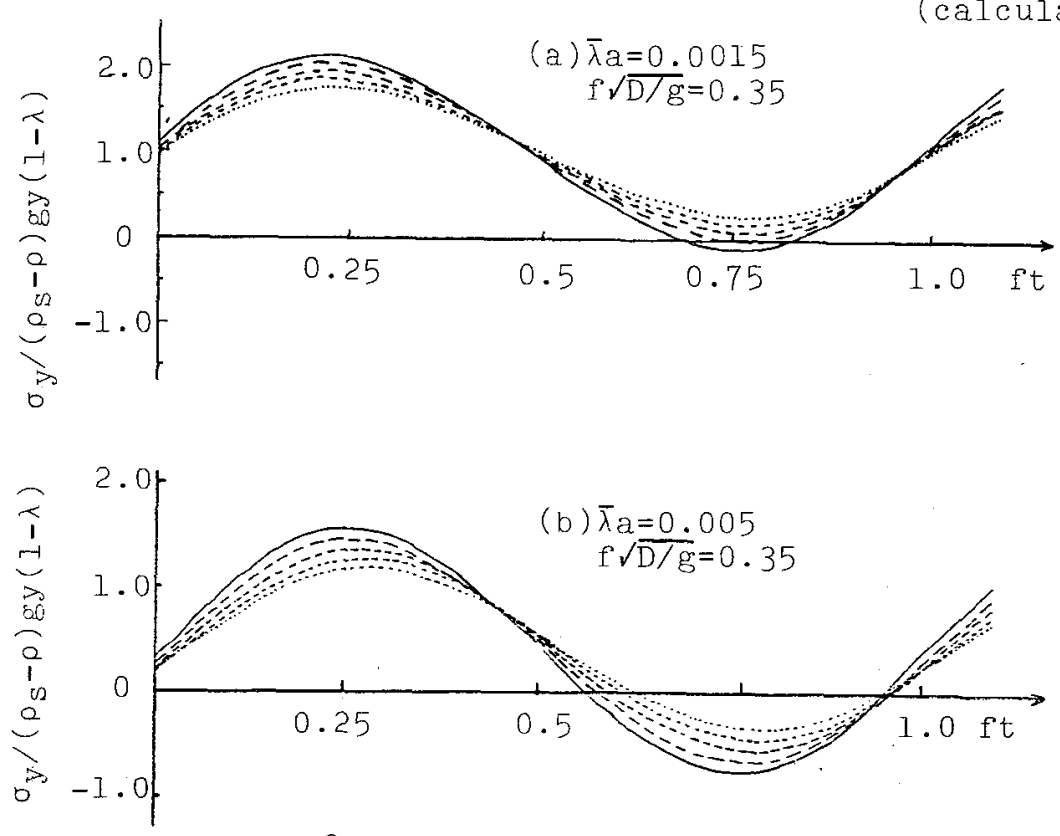

Fig. 8 Effective stress (calculated) 
follows.

1) on water : $\rho, \beta$

2) on sand layer : $\rho_{S}, \alpha, \lambda_{W}, \bar{\lambda}_{a}, k, D, \bar{h}$

3) on water pressure : $a_{0}, f, h_{0}$

4 ) on the field : $g, p_{0}$

Accordingly, the quantitative property $A$ of the liquefaction is given by the following functional relation.

$$
\begin{array}{r}
A=F\left(a_{0}, f, h_{0}, \rho_{S}, \alpha, \lambda_{W}, \bar{\lambda}_{a}, k, D,\right. \\
\left.\rho, \beta, g, p_{0}, \bar{h} ; y, t\right)
\end{array}
$$

By the dimensional analysis using $\rho, g$ and $D$ as the fundamental quantities of dimensions the above equation is transformed to,

$$
\begin{gathered}
\pi_{A}=\Phi\left(\frac{a_{0}}{D}, f \sqrt{\frac{D}{g}}, \frac{h_{0}}{D}, \frac{\rho_{S}}{\rho}, \alpha(\rho g D), \lambda_{W}, \bar{\lambda}_{a},\right. \\
\left.\frac{k}{\sqrt{g D}}, \beta(\rho g D), \frac{p_{0}}{\rho g D}, \frac{\bar{h}}{D} ; \frac{y}{D}, t \sqrt{\frac{g}{D}}\right)
\end{gathered}
$$

4.2 Maximum depth of liquified layer

Here we deal with the maximum depth of the liquified sand layer $y_{*}$ as one of the properties of liquefaction. It is analized theoretically under the following conditions,

$$
\rho_{\mathrm{S}} / \rho=2.65, \bar{h} / D=0.3, \mathrm{p}_{0} / \rho g D=10.3, \quad \beta(\rho \mathrm{gD})=0.41 \times 10^{-5} .
$$

In Figs. (9) to (14) the changes of the maximum depth $\mathrm{y}_{*}$ with $a_{0} / D, \bar{\lambda}_{a}, \bar{\lambda}_{W}, k / \sqrt{g D}, \alpha(\rho g D)$ and $h_{0} / D$ are shown with the parameter $f \sqrt{D / g}$.

From Figs. (9), (10) and (11) it is shown that the depth $y_{*}$ increases with the increase of $a_{0} / D, \bar{\lambda}_{a}$ and $\lambda_{w}$, and that the high frequency stimulates the liquefaction. Especialiy a very small amount of air in the sand affects the liquefaction considerably.

Fig.12 shows that $y_{*}$ decreases with the increase of the permeability. coefficient in the range beyond certain value of the coefficient.

Fig.13 shows that $y_{*}$ decreases rapidly with the increase 


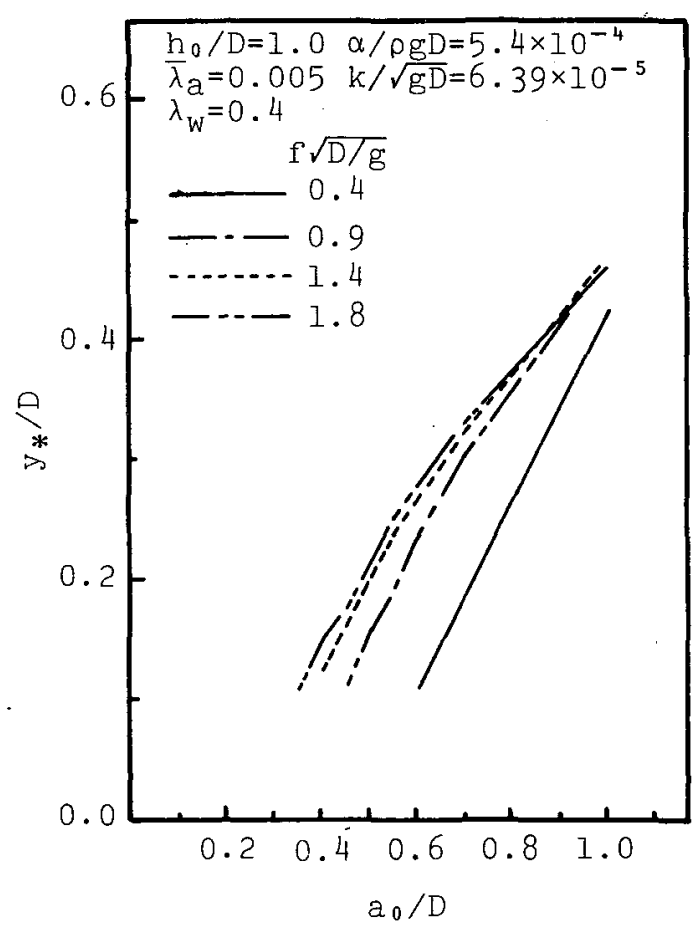

Fig. $9 \mathrm{a}_{0} / \mathrm{D} \sim \mathrm{y}_{*} / \mathrm{D}$

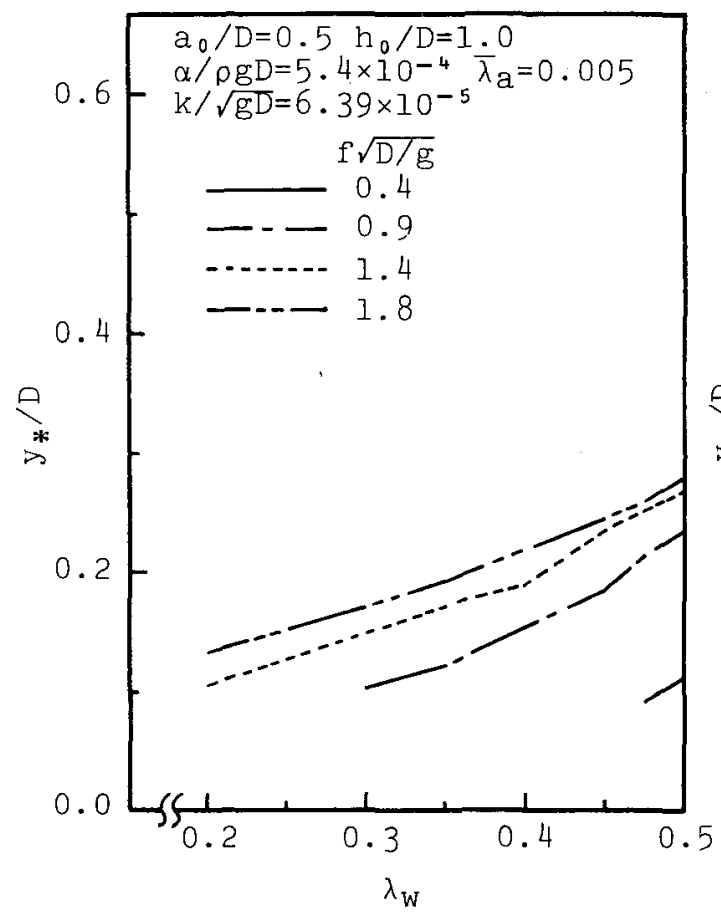

Fig. II $\lambda_{W} \sim y_{*} / D$

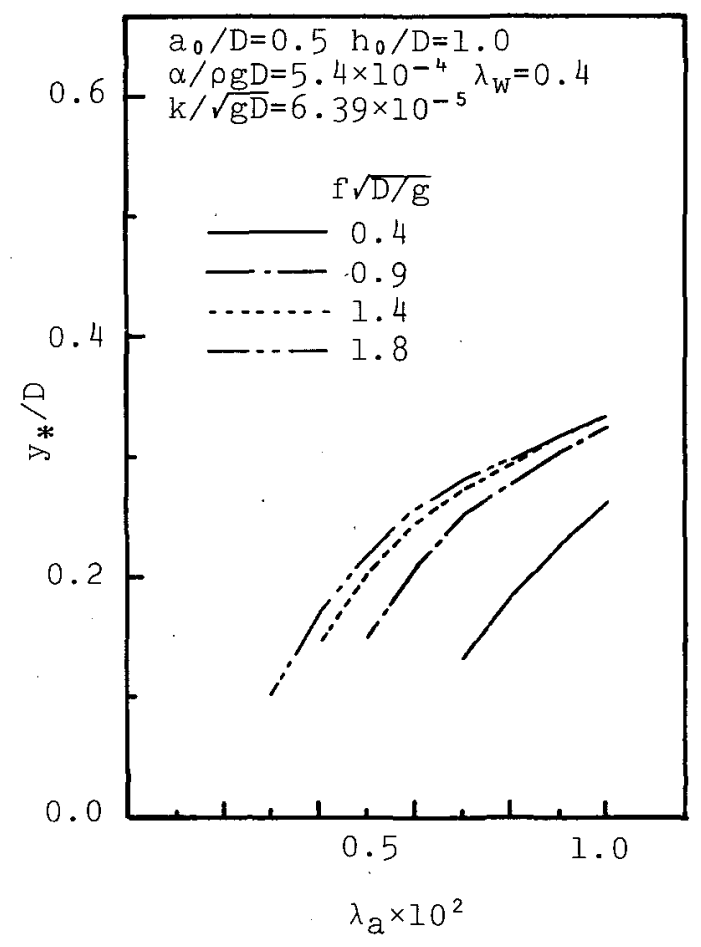

FIg.10 $\lambda_{a} \sim y_{*} / D$

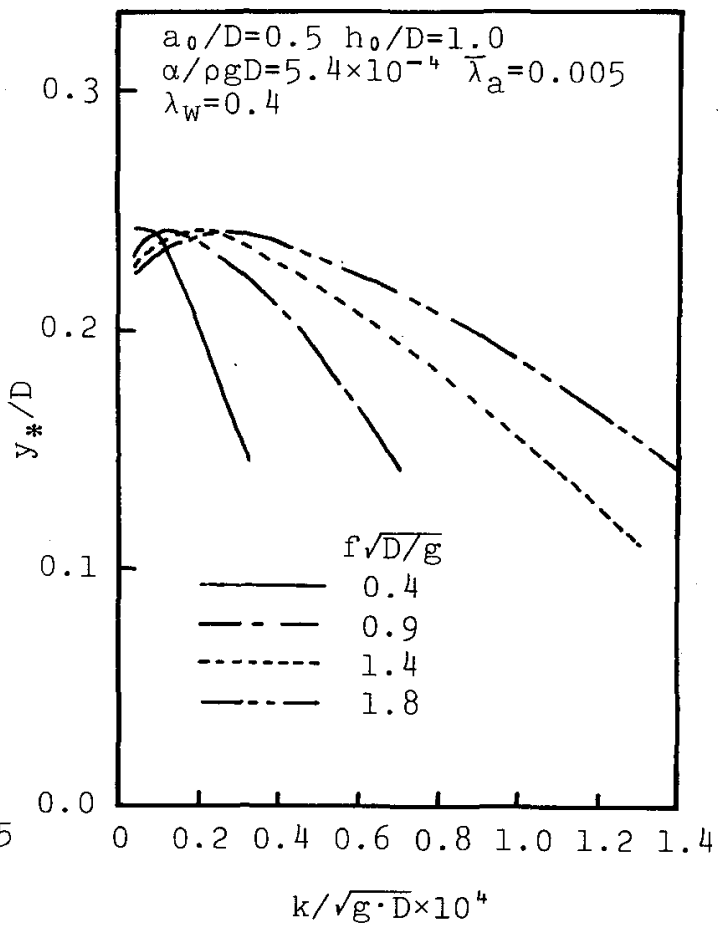

Fig. $12 \mathrm{k} / \sqrt{\mathrm{g} \cdot \overline{\mathrm{D}}} \sim \mathrm{y}_{*} / \mathrm{D}$ 


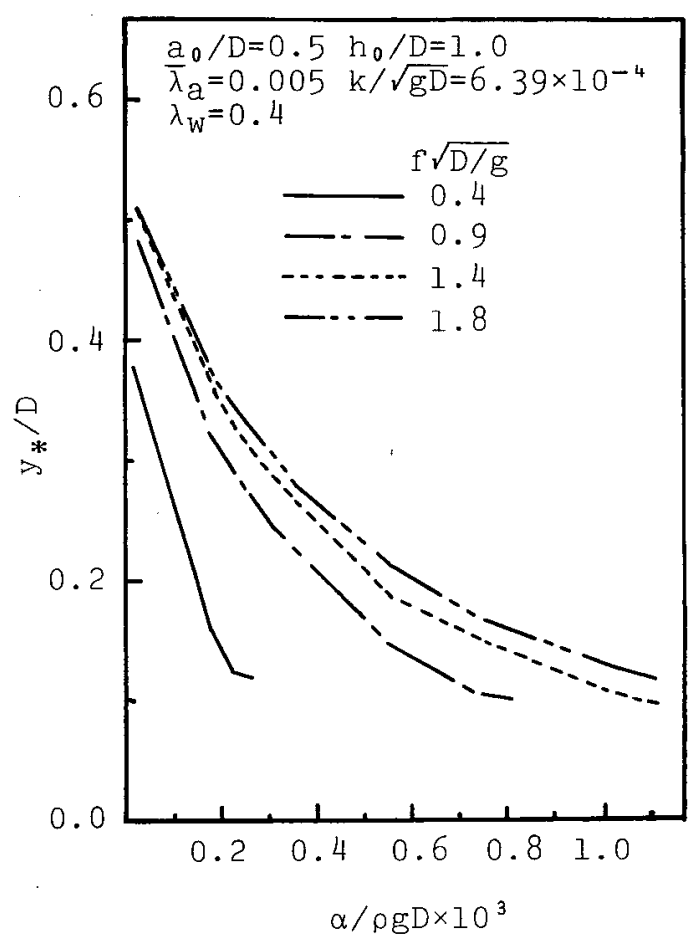

Fig. $13 \alpha / \rho g D \sim y_{*} / D$

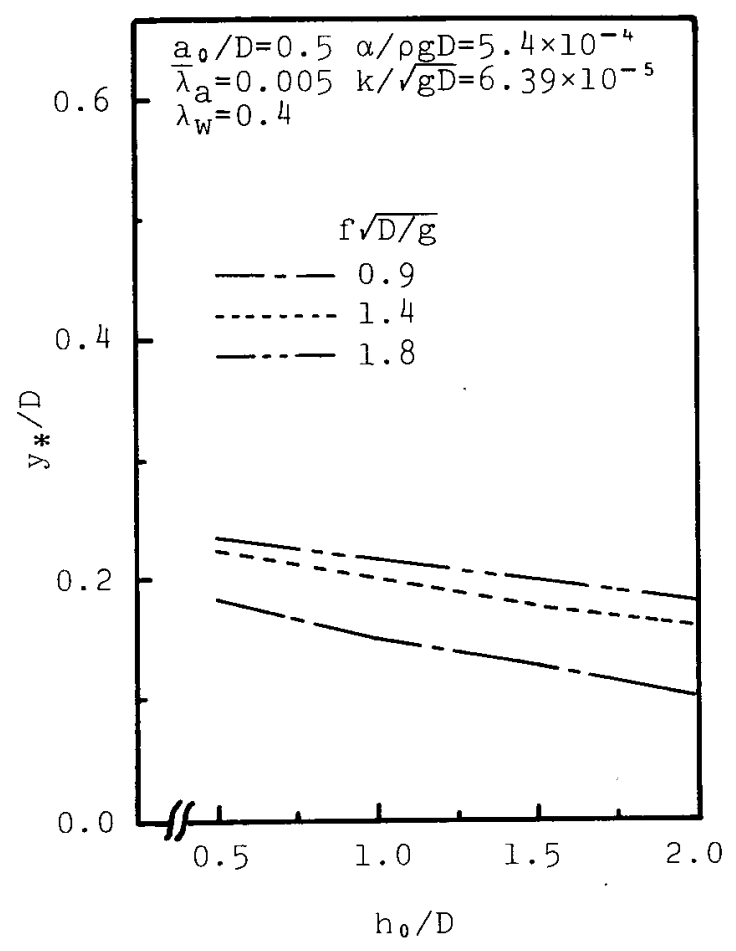

Fig. $14 h_{0} / D \sim y_{*} / D$

of the compressibility of the sand.

Fig. 14 shows that the increase of the mean water pressure decreases the liquified depth. But its degree is very small.

\section{Concluding remarks}

From the experiments and the theoretical analysis described above, we obtain following conclusions.

1) By the experiments it is clarified that the oscillating water pressure acting on the surface of the highly saturated sand layer propagates into the layer with the damping in amplitude and the lag in phase, and that under certain coditions the liquefaction appears. Further it becomes obvious that the damping ratio and the phase lag depend largely on the volume of the air involved in the layer.

These experimental results are explained fairly well by the theoretical analysis. 
2) By the theoretical analysis several properties on the liquefaction are obtained as follows.

The maximum depth of liquified layer increases with the increase of the amplitude and the frequency of the oscillating water pressure and the volume occupied by the air and the water in the sand layer. Especially a very small amount of the air affects the liquefaction properties significantly. The increase of the permeability coefficient in the range beyond certain value decreases the liquified depth. With the increase of the mean water pressure and the compressibility of the sand the liquified depth decreases. Especially in the small range of the compressibility the slope of the decreasing curve is very steep.

Acknowledgement

The author wishs to express his gratitude to Shuzo Furukawa, Akihiro Teraoka, Toshio Akura, Hiroshi Kawata and Másahiko Mitooka for their assistance in experiments and computations.

References

1) Hunter Rouse: Engineering Hydraulics, John Willey \& sons, New York, 1950, p.p. 326-331.

2) Reisaku Inoue: Propagation of pore water pressure in sand layer of high degree of saturation, Proc. JSCE, NNo. 236,1975 . 